\title{
Tamanho ótimo de parcela para avaliação da massa e diâmetro de cabeças de brócolis
}

\author{
Optimum plot size for evaluating the mass and diameter of broccoli heads \\ Betania Brum $^{I^{*}}$ Fernanda Daniela Brandelero ${ }^{\mathrm{I}}$ Thiago de Oliveira Vargas ${ }^{\mathrm{I}}$ \\ Lindolfo Storck ${ }^{I}$ II Pedro Paulo Gonçalves Zanini ${ }^{I}$
}

\section{RESUMO}

$O$ objetivo deste trabalho foi determinar o tamanho ótimo de parcela $(X o)$, por amostragem na área total e nas frações de áreas, a fim de avaliar a massa e o diâmetro de cabeças de brócolis. Em campo, foram demarcadas 27 filas do híbrido BRO $68^{\circledR}$. Cada planta foi considerada como sendo uma unidade experimental básica (UEB). Para fins de análise, cada fila foi considerada como sendo um ensaio em branco com 100 UEB. Para os resultados das 27 filas, obtiveram-se os valores: mínimo, máximo, média, desvio padrão, $\mathrm{CV}$, intervalo de confiança, o tamanho de amostra (número de filas) para estimar o Xo e para estimar a média. O tamanho ótimo de parcela para avaliar a massa e diâmetro de cabeças de brócolis é igual a cinco unidades experimentais básicas (plantas).

Palavras-chave: Brassica oleracea var. italica, variação espacial, amostragem, planejamento experimental.

\section{ABSTRACT}

The objective of this study was to determine the optimum plot size by sampling the total area and the fractions areas in order to evaluate the mass and diameter of broccoli heads. In the field, 27 rows of hybrid $B R O 68^{\circledR}$ were demarcated. Each plant was considered as an experimental basic unit (UEB). For analysis purposes, each row was regarded as being one blank test on 100 UEB. For the results of 27 rows yielded values: minimum, maximum, average, standard deviation, $C V$, confidence interval, the sample size (number of rows) to estimate Xo and to estimate the mean. The optimum plot size to evaluate the mass and diameter of broccoli heads equals five basic experimental units (plants).

Key words: Brassica oleracea var. italica, spatial variation sampling, experimental design.

\section{INTRODUÇÃO}

O brócolis (Brassica oleracea L. var. italica Plenck) é uma ótima fonte de nutrientes, além disso, o consumo regular previne alguns tipos de câncer, doenças cardíacas e purifica o sangue. Mesmo com todos os nutrientes que oferece, é um alimento pobre em calorias, sendo indicado em dietas de perda de peso (ROSA \& RODRIGUES, 2001). Além disso, é uma hortaliça rica em Boro (B), que atua no metabolismo de substratos energéticos, no funcionamento do cérebro e na atividade psicomotora e cognitiva (PIZETTA et al., 2005).

Para obter melhor produtividade e qualidade de brócolis, vêm sendo realizadas pesquisas referentes às adubações, aos manejos, incidência de doenças (DINIZ, 2008; CAMPAGNOL et al., 2009; TREVISAN et al., 2003), entre outros, sem o conhecimento correto do planejamento experimental. Nesse sentido, o conhecimento da variabilidade da produção, numa área experimental, é importante para o planejamento adequado de experimentos. $\mathrm{O}$ planejamento define, para um determinado número de tratamentos, o delineamento experimental, o tamanho da parcela e o número de repetições (RAMALHO et al., 2000; STORCK et al., 2011).

Para conhecer a variabilidade na área experimental, podem ser conduzidos ensaios em branco, específicos para a identificação da

\footnotetext{
'Departamento de Ciências Agrárias, Universidade Tecnológica Federal do Paraná (UFTPR) 85503-390, Pato Branco, PR, Brasil. E-mail: bbufsm@gmail.com. *Autor para correspondência.

"Programa de Pós-graduação em Agronomia, Universidade Federal de Santa Maria (UFSM), Santa Maria, RS, Brasil. Recebido 22.02.15 Aprovado 23.06.15 Devolvido pelo autor 23.10.15 CR-2015-0236.R2
} 
variabilidade ou do índice de heterogeneidade da característica avaliada. Nessa metodologia, são coletados os dados da produção de pequenas parcelas denominadas de unidades experimentais básicas (UEB) e, com estas UEB, são planejadas parcelas de diferentes tamanhos, pelo agrupamento de UEB adjacentes. Para cada tamanho de parcela $(X)$, são calculados os coeficientes de variação $(\mathrm{CVx})$. Da relação $\mathrm{CVx}=\mathrm{A} / \mathrm{X}^{\mathrm{B}}$ é estimado o tamanho ótimo de parcela (Xo) como sendo o ponto de máxima curvatura dessa relação (MEIER \& LESSMAN, 1971), sendo A e B os parâmetros da equação. No entanto, a dificuldade e o trabalho em planejar diferentes tamanhos de parcelas pelo agrupamento de UEB adjacentes, no método de máxima curvatura, foram superados com a aplicação do método da curvatura máxima do modelo do coeficiente de variação (PARANAÍBA et al., 2009a). Nesse método, a partir dos dados das UEB de um ensaio em branco, é estimado o coeficiente de autocorrelação espacial de primeira ordem, a variância e a média, usados para o cálculo do tamanho ótimo de parcela. Este método tem sido adequado para a culturas de milho (CARGNELUTTI FILHO et al., 2011a), arroz (PARANAÍBA et al., 2009a), trigo e mandioca (PARANAÍBA et al., 2009b).

A possibilidade, para algumas espécies cultivadas em filas quase independentes (mandioca, batata, couve, brócolis, etc), de se usar os dados de algumas linhas ou grupos de linhas com determinado comprimento, de forma aleatória sobre toda a área experimental, é uma alternativa viável, segundo estudos na cultura da batata (STORCK, 2011), resultando em economia de recursos financeiros e humanos. A determinação do tamanho de parcela por amostragem de quadros menores na área experimental é favorecida pela facilidade de aplicação do método de PARANAÍBA et al. (2009a).

Usualmente, em experimentos com brócolis, foram usadas parcelas de 10 plantas na comparação da produtividade de cultivares (LALLA et al., 2010), 14 plantas na avaliação de cultivares em plantio direto sobre diferentes culturas de cobertura (MELO et al., 2010) e 14 plantas (10 úteis) na avaliação de cultivares (TREVISAN et al., 2003). No entanto, o tamanho ótimo de parcela e o número de ensaios (quadros) necessários para estimativa do tamanho ótimo de parcela em couve e em brócolis não são conhecidos. Assim, os objetivos deste trabalho foram determinar o tamanho ótimo de parcela por amostragem na área total e nas frações de áreas, a fim de avaliar a massa e o diâmetro de cabeças de brócolis.

\section{MATERIAL E MÉTODOS}

O experimento foi conduzido na área experimental da UTFPR em Pato Branco, PR. A área está situada na latitude $26^{\circ} 06^{\prime} 59^{\prime \prime} \mathrm{S}$, longitude $52^{\circ}$ 40' 59'W, e com altitude de 721,80m. O município de Pato Branco apresenta clima tipo "cfa" subtropical úmido, com chuvas bem distribuídas ao longo do ano, temperatura média no mês mais frio inferior a $18^{\circ} \mathrm{C}$, temperatura média no mês mais quente acima de $22^{\circ} \mathrm{C}$, com verões relativamente quentes. Foram demarcadas 27 filas de 50 metros de comprimento, espaçadas em $0,80 \mathrm{~m}$, resultando em $1080 \mathrm{~m}^{2}$ de área. A bordadura da área foi de, aproximadamente, quatro metros em todos os lados. Nessa área, foram plantadas mudas de brócolis com $0,50 \mathrm{~m}$ entre plantas. As mudas de brócolis foram produzidas em estufa usando sementes do híbrido BRO $68^{\circledR}$ e substrato Tecnomax ${ }^{\circledR}$, preparadas em bandejas de poliestireno expandido de 128 células. Aos 46 dias após a semeadura, foram transplantadas para a área experimental demarcada.

Antes do plantio, a área foi preparada com subsolagem e enxada rotativa. A adubação de base foi realizada nas filas com $87,5 \mathrm{~kg} \mathrm{ha}^{-1}$ de $\mathrm{N}$ (ureia), $1000 \mathrm{~kg} \mathrm{ha}^{-1}$ de $\mathrm{P}_{2} \mathrm{O}_{5}$ (superfosfato simples) e $25 \mathrm{~kg}$ $\mathrm{ha}^{-1}$ de $\mathrm{KCl}$ (cloreto de potássio). Aos 20 e aos 44 dias após o transplante, foram realizadas adubações de cobertura com $87,5 \mathrm{~kg} \mathrm{ha}^{-1}$ de ureia e $25 \mathrm{~kg} \mathrm{ha}^{-1}$ de $\mathrm{KCl}$. Na primeira adubação de cobertura, o adubo foi incorporado superficialmente, realizando-se também a amontoa para melhorar, principalmente, a sustentação das plantas.

Foram realizadas três aplicações foliares dos micronutrientes Boro (B) e Molibdênio (Mo), em quantidades suficientes para completo molhamento foliar e concentração de ácido bórico $\left(1 \mathrm{~g} \mathrm{~L}^{-1}\right)$ e molibdato de sódio $\left(0,5 \mathrm{~g} \mathrm{~L}^{-1}\right)$. A primeira aplicação foi realizada na fase de mudas, e, as outras, realizadas aos 15 e 30 dias após o transplantio.

No ponto de colheita, as cabeças foram cortadas e imediatamente avaliadas para evitar a perda de água por desidratação. Foram determinados diâmetro $(\mathrm{cm})$ e massa $(\mathrm{g})$, anotando a posição em que estavam no campo (fila e coluna). A posição de campo foi identificada pelo número de ordem da fila (1 a 27) e do número de ordem da coluna (1 a 100), formando uma matriz de 2.700 observações.

Cada planta foi considerada como sendo uma unidade experimental básica (UEB). Para fins de análise, cada fila foi considerada como sendo um ensaio em branco (ensaio de uniformidade) com 100 UEB. Para cada 
ensaio e para cada um dos caracteres (massa e diâmetro), foi estimada a média $(\mathrm{m})$, a variância $\left(s^{2}\right)$ e o coeficiente de variação $(\mathrm{CV})$ entre as plantas. Também foi estimado o coeficiente de autocorrelação espacial de primeira ordem ( $\hat{\rho}$ ), usando a sequência de numeração das plantas dentro de cada fila. Sendo $X_{i}$ igual ao valor observado na cabeça i $(\mathrm{i}=1,2, \ldots \mathrm{n}=100)$, temse: $\mathrm{D}_{\mathrm{i}}=\mathrm{X}_{\mathrm{i}}-\mathrm{m}$ e $\hat{\rho}=\sum_{i=2}^{n=100} D_{i} D_{i-1} / \sum_{i=1}^{n=100} D_{i}^{2}$, segundo PARANAÍBA et al. (2009a). Conforme os mesmos autores, o tamanho ótimo da parcela (Xo, número de plantas adjacentes) por fila foi estimado por meio do método da curvatura máxima do modelo do coeficiente de variação (CMMCV) pela expressão $X o=(10 / m)\left(2\left(1-\hat{\rho}^{2}\right) s^{2} m\right)^{1 / 3}$, sendo que o coeficiente de variação entre as parcelas de tamanho $X o$ foi estimado pela expressão $C V_{X o}=100 \sqrt{\left(1-\hat{\rho}^{2}\right) s^{2} / m^{2}} / \sqrt{X o}$.

Para os resultados obtidos nas 27 filas (ensaios), obtiveram-se os valores: mínimo, máximo, média, desvio padrão, $\mathrm{CV}$, intervalo de confiança $(1-\alpha=0,95)$, o tamanho de amostra (número de filas) para estimar o Xo (com erro de estimação equivalente a amplitude de uma UEB) e para estimar a média (com erro de estimação igual a $10 \%$ da média do caractere), com base na distribuição $\mathrm{t}(\alpha=0,05)$. A normalidade dos dados observados de Xo e da média $(\mathrm{m})$ foi verificada pelo aplicativo WinSTAT ${ }^{\circledR}$ for Microsoft ${ }^{\circledR}$ Excel. Para os demais procedimentos, foi usado o software R (R DEVELOPMENT CORE TEAM, 2013) e o aplicativo Microsoft Office Excel.

A área de teste (2.700 UEB) foi dividida em três quadros, com as filas variando entre 1 a 27 e as colunas entre 01 a 33 (quadro 1), entre 34 e 67 (quadro 2) e entre 68 e 100 (quadro 3). Para cada fila, dentro de cada quadro com 33 (891 UEB) ou 34 (918 UEB) colunas, foram estimadas as estatísticas: $\mathrm{m}, \mathrm{s}^{2}, \mathrm{CV}, \hat{\rho}, X o$ e $C V_{X o}$.

Dentro de cada quadro, foi estimado o tamanho de amostra (número de filas) para estimar o Xo (com erro de estimação igual a uma planta), com base na distribuição $t(\alpha=0,05)$.

\section{RESULTADOS E DISCUSSÃO}

A média geral da massa por cabeça de brócolis foi igual a $1113 \mathrm{~g}$. Considerando o espaçamento $(0,5 \times 0,8 \mathrm{~m})$, a produtividade foi igual a $27,8 \mathrm{t} \mathrm{ha}^{-1}$. Este resultado é superior ao obtido em experimento de comparação de cultivares, máximo de $15,4 \mathrm{t} \mathrm{ha}^{-1}$ para a cultivar AF649 (LALLA et al.,
2010), máximo de 12,5 h ha $^{-1}$ para adubação orgânica de 25t ha-1 (DINIZ et al., 2008), 13,2t ha ${ }^{-1}$ (MELO et al., 2010) e 16,3t ha-1 (TREVISAN et al., 2003).

As plantas com falhas ( $4,7 \%$ das plantas), ou seja, com ausência de produção de cabeça de brócolis, motivado por danos mecânicos, doenças e pragas, foram substituídas pela média das duas plantas vizinhas. Normalmente, em experimentação, havendo perda de plantas não causada pelo efeito de tratamento, solução semelhante é adotada, ou seja, é obtida a média das unidades presentes (STORCK et al., 2011). Portanto, pela produtividade obtida e a baixa frequência de falhas, os dados obtidos são adequados para o estudo proposto.

A área experimental não é homogênea, fato que pode ser observado na figura 1 (massa de cabeça e diâmetro de cabeça). Nesta figura, quando LS de uma fila é menor que LI de outra fila, estas filas diferem significativamente $(\alpha<0,05)$ pelo teste t. Dessa forma, observam-se diferenças entre

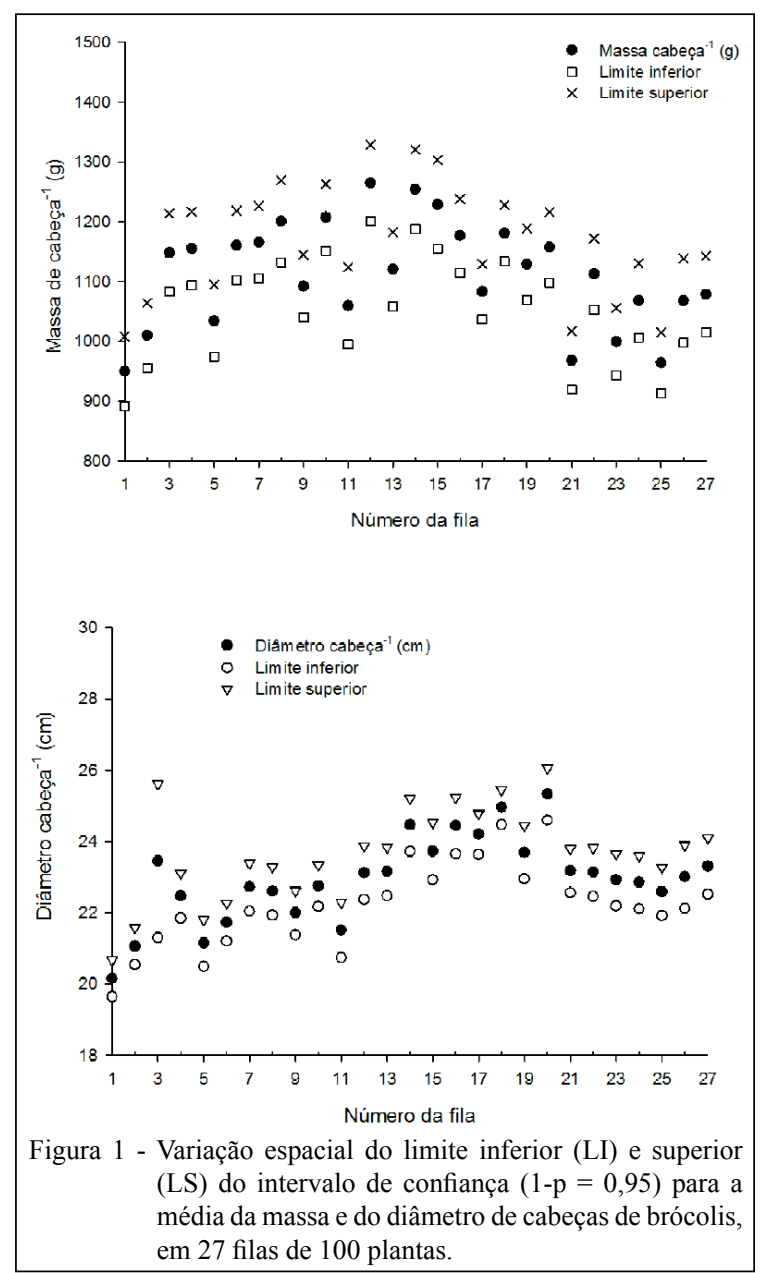

Ciência Rural, v.46, n.3, mar, 2016. 
diversas filas quanto aos dois caracteres (massa e diâmetro). A justificativa para tais diferenças está nas condições iniciais de fertilidade da área e na introdução de variação, devido à adubação e manejo que foram executados por fila. Constatação semelhante foi relatada, anteriormente, com a cultura da batata (OLIVEIRA et al., 2005), possivelmente pelas mesmas razões. Nesses casos, a solução é adotar o delineamento blocos ao acaso, em que os blocos são formados pelas filas, ou seja, cada bloco é um grupo de unidades experimentais homogêneas (ZIMMERMANN, 2004; RAMALHO et al., 2005).

As estimativas da média $(\mathrm{m}), \mathrm{s}^{2}, \mathrm{CV}$, $\hat{\rho}, X o$ e $C V_{X o}$ estão relatadas na tabela 1 (massa de cabeça) e tabela 2 (diâmetro de cabeça). O tamanho ótimo de parcela para a estimativa da massa de cabeça de brócolis é igual a 5,24 UEB (cinco plantas), variando entre os limites de 5,09 e 5,38 plantas, com $95 \%$ de confiança. O tamanho

Tabela 1 - Média da massa $\left(\mathrm{g}\right.$ cabeça $\left.{ }^{-1}\right)$ de brócolis, variância $\left(\mathrm{s}^{2}\right)$, coeficiente de variação $(\mathrm{CV})$, coeficiente de autocorrelação espacial $(\hat{\rho})$, tamanho ótimo de parcela $(X o)$ e $\mathrm{CV}$ para $X o\left(\mathrm{CV}_{\mathrm{Xo}}\right)$, para as diferentes filas da área de teste.

\begin{tabular}{|c|c|c|c|c|c|c|}
\hline Fila & Média & $s^{2}$ & $\mathrm{CV}$ & $\hat{\rho}$ & $X o$ & $\mathrm{CV}_{\mathrm{Xo}}$ \\
\hline 1 & 950 & 86113 & 30,89 & 0,135 & 5,72 & 12,80 \\
\hline 2 & 1010 & 74379 & 27,00 & 0,063 & 5,26 & 11,75 \\
\hline 3 & 1148 & 108817 & 28,73 & 0,418 & 5,15 & 11,51 \\
\hline 4 & 1155 & 97160 & 26,98 & 0,283 & 5,12 & 11,44 \\
\hline 5 & 1035 & 93099 & 29,49 & 0,114 & 5,56 & 12,43 \\
\hline 6 & 1160 & 86848 & 25,40 & $-0,064$ & 5,05 & 11,28 \\
\hline 7 & 1166 & 93408 & 26,21 & 0,077 & 5,15 & 11,52 \\
\hline 8 & 1201 & 118692 & 28,69 & $-0,121$ & 5,45 & 12,19 \\
\hline 9 & 1092 & 70884 & 24,37 & $-0,042$ & 4,91 & 10,99 \\
\hline 10 & 1208 & 80062 & 23,43 & 0,057 & 4,78 & 10,70 \\
\hline 11 & 1059 & 107176 & 30,91 & $-0,212$ & 5,67 & 12,68 \\
\hline 12 & 1265 & 103823 & 25,47 & 0,105 & 5,04 & 11,28 \\
\hline 13 & 1120 & 97687 & 27,89 & $-0,214$ & 5,30 & 11,84 \\
\hline 14 & 1254 & 112680 & 26,76 & $-0,197$ & 5,16 & 11,55 \\
\hline 15 & 1229 & 139451 & 30,38 & $-0,233$ & 5,59 & 12,50 \\
\hline 16 & 1177 & 96527 & 26,40 & 0,150 & 5,15 & 11,51 \\
\hline 17 & 1083 & 54572 & 21,57 & 0,035 & 4,53 & 10,13 \\
\hline 18 & 1181 & 56924 & 20,20 & 0,093 & 4,32 & 9,67 \\
\hline 19 & 1129 & 91040 & 26,72 & $-0,057$ & 5,22 & 11,68 \\
\hline 20 & 1158 & 89088 & 25,79 & $-0,090$ & 5,09 & 11,38 \\
\hline 21 & 968 & 60824 & 25,48 & 0,170 & 5,01 & 11,21 \\
\hline 22 & 1113 & 90884 & 27,10 & $-0,054$ & 5,27 & 11,79 \\
\hline 23 & 1000 & 80981 & 28,47 & 0,023 & 5,45 & 12,19 \\
\hline 24 & 1068 & 99160 & 29,48 & 0,086 & 5,57 & 12,45 \\
\hline 25 & 964 & 66315 & 26,71 & $-0,126$ & 5,20 & 11,62 \\
\hline 26 & 1068 & 127025 & 33,37 & 0,084 & 6,05 & 13,52 \\
\hline 27 & 1079 & 103405 & 29,81 & 0,059 & 5,62 & 12,56 \\
\hline Mínimo & 950 & 54572 & 20,20 & $-0,233$ & 4,32 & 9,67 \\
\hline Máximo & 1265 & 139451 & 33,37 & 0,418 & 6,05 & 13,52 \\
\hline Média & 1113 & 92112 & 27,17 & 0,020 & 5,24 & 11,71 \\
\hline DP & 88 & 20466 & 2,88 & 0,154 & 0,37 & 0,82 \\
\hline $\mathrm{CV}$ & 7.9 & 22,2 & 10,6 & - & 7,0 & 7,0 \\
\hline LI* & 1078 & 84016 & 26,03 & $-0,041$ & 5,09 & 11,38 \\
\hline LS & 1147 & 100208 & 28,31 & 0,081 & 5,38 & 12,03 \\
\hline $\mathrm{n}_{\mathrm{o}(\mathrm{e}=10 \%)}$ & 2.6 & & & & & \\
\hline $\mathrm{n}_{\mathrm{o}(\mathrm{e}=1 \text { UEB })}$ & & & & & 0,6 & \\
\hline
\end{tabular}

Limite inferior (LI) e superior (LS) do intervalo de confiança $(1-\alpha=0,95)$ e tamanho de amostra $\left(\mathrm{n}_{\mathrm{o}}, \alpha=0,05\right)$ para erro de estimação de $10 \%$ da média (da massa) e erro de uma UEB em Xo.

Ciência Rural, v.46, n.3, mar, 2016. 
Tabela 2 - Média do diâmetro (cm) de cabeça de brócolis, variância $\left(\mathrm{s}^{2}\right)$, coeficiente de variação (CV), coeficiente de autocorrelação espacial $(\hat{\rho})$, tamanho ótimo de parcela $(X o)$ e $\mathrm{CV}$ para $X o\left(\mathrm{CV}_{\mathrm{Xo}}\right)$ para as diferentes filas da área de teste.

\begin{tabular}{|c|c|c|c|c|c|c|}
\hline Fila & Média & $s^{2}$ & $\mathrm{CV}$ & $\hat{\rho}$ & $X o$ & $\mathrm{CV}_{\mathrm{Xo}}$ \\
\hline 1 & 20,18 & 6,72 & 12,85 & 0,090 & 3,20 & 7,15 \\
\hline 2 & 21,09 & 6,75 & 12,32 & 0,027 & 3,12 & 6,97 \\
\hline 3 & 22,48 & 10,25 & 14,24 & 0,492 & 3,13 & 7,01 \\
\hline 4 & 22,50 & 10,11 & 14,14 & 0,395 & 3,23 & 7,22 \\
\hline 5 & 21,17 & 11,00 & 15,67 & 0,073 & 3,66 & 8,17 \\
\hline 6 & 21,75 & 7,11 & 12,26 & $-0,013$ & 3,11 & 6,95 \\
\hline 7 & 22,74 & 11,56 & 14,95 & 0,155 & 3,52 & 7,87 \\
\hline 8 & 22,63 & 11,68 & 15,10 & 0,100 & 3,56 & 7,96 \\
\hline 9 & 22,02 & 9,77 & 14,20 & 0,226 & 3,37 & 7,54 \\
\hline 10 & 22,76 & 8,52 & 12,82 & 0,306 & 3,10 & 6,93 \\
\hline 11 & 21,53 & 15,25 & 18,14 & $-0,091$ & 4,03 & 9,00 \\
\hline 12 & 23,14 & 14,39 & 16,40 & 0,248 & 3,70 & 8,26 \\
\hline 13 & 23,18 & 11,87 & 14,86 & $-0,017$ & 3,54 & 7,90 \\
\hline 14 & 24,48 & 13,99 & 15,27 & $-0,122$ & 3,58 & 8,01 \\
\hline 15 & 23,75 & 16,59 & 17,15 & $-0,097$ & 3,88 & 8,67 \\
\hline 16 & 24,46 & 15,90 & 16,30 & 0,252 & 3,68 & 8,22 \\
\hline 17 & 24,24 & 8,27 & 11,86 & 0,278 & 2,96 & 6,62 \\
\hline 18 & 24,98 & 6,05 & 9,85 & 0,338 & 2,58 & 5,77 \\
\hline 19 & 23,72 & 14,17 & 15,87 & 0,097 & 3,68 & 8,23 \\
\hline 20 & 25,35 & 13,67 & 14,58 & 0,198 & 3,44 & 7,70 \\
\hline 21 & 23,20 & 9,69 & 13,42 & 0,281 & 3,21 & 7,19 \\
\hline 22 & 23,16 & 11,93 & 14,91 & 0,087 & 3,53 & 7,90 \\
\hline 23 & 22,94 & 13,63 & 16,09 & 0,063 & 3,72 & 8,32 \\
\hline 24 & 22,88 & 14,15 & 16,44 & $-0,008$ & 3,78 & 8,46 \\
\hline 25 & 22,61 & 11,66 & 15,10 & $-0,085$ & 3,56 & 7,97 \\
\hline 26 & 23,04 & 20,16 & 19,49 & 0,060 & 4,23 & 9,46 \\
\hline 27 & 23,33 & 15,71 & 16,99 & 0,129 & 3,84 & 8,59 \\
\hline Mínimo & 20,18 & 6,05 & 9,85 & $-0,122$ & 2,58 & 5,77 \\
\hline Máximo & 25,35 & 118,38 & 46,34 & 0,395 & 7,54 & 16,87 \\
\hline Média & 22,97 & 15,88 & 16,05 & 0,111 & 3,64 & 8,15 \\
\hline DP & 1,20 & 20,77 & 6,40 & 0,144 & 0,86 & 1,91 \\
\hline $\mathrm{CV}$ & 5,2 & 130,8 & 39,9 & - & 23,5 & 23,5 \\
\hline LI & 22,50 & 7,66 & 13,52 & 0,054 & 3,30 & 7,39 \\
\hline LS & 23,45 & 24,09 & 18,58 & 0,167 & 3,98 & 8,90 \\
\hline $\mathrm{n}_{\mathrm{o}(\mathrm{e}=10 \%)}$ & 1,1 & & & & & \\
\hline $\mathrm{n}_{\mathrm{o}(\mathrm{e}=1 \mathrm{UEB})}$ & & & & & 3,1 & \\
\hline
\end{tabular}

${ }^{*}$ Limite inferior (LI) e superior (LS) do intervalo de confiança $(1-\alpha=0,95)$ e tamanho de amostra (no, $\left.\alpha=0,05\right)$ para erro de estimação de $10 \%$ da média (do diâmetro) e erro de uma UEB em Xo.

ótimo de amostra para a variável diâmetro de cabeça é menor do que o necessário para massa de cabeça, mas é igualmente homogêneo entre as 27 filas (Tabela 2). Esses resultados são inferiores ao tamanho de parcela tradicionalmente utilizado na avaliação da produção de brócolis (TREVISAN et al., 2003; LALLA et al., 2010; MELO et al., 2010).

O coeficiente de autocorrelação espacial de primeira ordem $(\hat{\rho})$, no caso do caractere massa, é baixo e não difere significativamente $(1-\alpha=0,95)$ de zero, o intervalo de confiança contém o valor zero. Com isso, existe uma independência espacial entre as UEB da área experimental, propriedade importante para os pressupostos dos modelos dos delineamentos experimentais. Para o caractere diâmetro de cabeça, apesar do $\hat{\rho}$ ser significativamente maior do que zero, a amplitude de variação $(0,054<\rho<0,167 ; 1-\alpha=0,95)$ é pequena (Tabela 2). 
Observando os valores de LI e LS de $\hat{\rho}$, constata-se que, para o caractere diâmetro de cabeça, a autocorrelação $(\hat{\rho})$ é significativamente maior que zero, pois os limites entre LI $(=0,054)$ e $\operatorname{LS}(=0,167)$ não contém o valor zero. Considerando que o método para estimar Xo não depende desta significância, não vemos restrições de validade dos resultados. As estimativas de $\mathrm{m}$ e $X o$, referentes as 27 filas, se ajustam à distribuição normal, constatada pelos testes de Kolmogorov-Smirnov $(\alpha>0,60)$. Com isso, é possível determinar o tamanho de amostra (número de filas) usando a distribuição t. Para estimar o caractere massa (g) por cabeça de brócolis, é suficiente avaliar três fileiras de 100 plantas, considerando um erro de estimação de $10 \%$ da média. Com aproximadamente o mesmo erro de estimação, para avaliar o diâmetro da cabeça, é necessário avaliar apenas uma fila (Tabela 2). O tamanho de amostra (número de filas) para estimar o tamanho ótimo de parcela, usando o valor máximo dos dois caracteres (massa e diâmetro) é igual a três filas com erro de estimação equivalente a uma amplitude de uma UEB (menor erro possível) (Tabela 2). Dessa forma, numa área experimental, amostrar três filas de 100 plantas é o suficiente para estimar Xo (com erro de estimação equivalente a uma UEB), visto que existe um padrão de variabilidade dentro das filas.

A prática de amostragem de partes da área experimental (pequenos lotes) para a determinação do tamanho ótimo de parcela também foi relatada para ensaios de batata (STORCK, 2011), como indicativo para a redução dos recursos humanos. Dessa forma, é possível sugerir que a estimativa do tamanho ótimo de parcela, referente a uma área experimental grande, pode ser realizada a partir da amostragem de alguns lotes menores dentro da área maior. A média dos tamanhos ótimos desses lotes é válida para a área total.
Ao dividir a área experimental em três quadros (Tabela 3 ), as médias das estimativas do $\hat{\rho}$ e de $X o$ referentes aos diferentes quadros também são homogêneos e semelhantes aos obtidos com 27 filas de 100 plantas. O tamanho de amostra (número de filas) aumentou, praticamente, na proporção inversa da redução do tamanho do ensaio em branco. Assim, ao usar 27 filas de 100 plantas, são necessárias 2,3 filas ou 230 plantas $(230 / 2700$ ou $8,5 \%$ da área total $)$ e, ao usar 27 filas de 33 ou 34 plantas, são necessárias 9,12 filas ou 297 plantas $(297 / 2700=11,0 \%$ da área total). Na condição de ensaios de 33 ou 34 plantas, observa-se maior variabilidade entre as estimativas de $X o$ e por isso o tamanho de amostra é maior. Este fato corrobora com o reportado por CARGNELUTTI FILHO et al. (2011b), que observaram relação entre o tamanho dos ensaios e a variação do tamanho ótimo de parcelas em estudo de simulação com dados de massa fresca de nabo forrageiro. Portanto, o tamanho do ensaio em branco não pode ser tão pequeno, porque aumenta a amplitude de variação do tamanho de parcela e de outras estatísticas.

Em geral, a estratégia de amostrar "quadros" menores, dentro de uma futura área experimental, é apropriada para a determinação do plano experimental, quanto ao tamanho de parcela, número de repetições e delineamento experimental. Essa afirmação está amparada nos resultados deste estudo e nos resultados com ensaio de uniformidade de batata (STORCK, 2011 ), em que $39 \%$ dos quadros menores, dentro da área total, foram suficientes para determinar o tamanho ótimo de parcela.

\section{CONCLUSÃo}

O tamanho ótimo de parcela para avaliar a massa e diâmetro de cabeças de brócolis é igual a cinco unidades experimentais básicas (plantas).

Tabela 3 - Média do coeficiente de autocorrelação espacial $(\hat{\rho})$ e do tamanho ótimo de parcela $(X o)$ e tamanho de amostra (número de filas, $\left.\mathrm{n}_{\mathrm{o}(\mathrm{Xo})}\right)$ para estimar Xo na área de teste de produção de brócolis.

\begin{tabular}{|c|c|c|c|c|c|c|c|}
\hline \multirow{2}{*}{ Quadro } & \multirow{2}{*}{ Colunas } & \multirow[b]{2}{*}{$\hat{\rho}$} & \multirow[b]{2}{*}{ Xo } & \multirow[b]{2}{*}{$\mathrm{n}_{\mathrm{o}(\mathrm{Xo})}$} & \multirow[b]{2}{*}{$\hat{\rho}$} & \multirow[b]{2}{*}{ Xo } & \multirow[b]{2}{*}{$\mathrm{n}_{\mathrm{o}(\mathrm{Xo})}$} \\
\hline & & & & & & & \\
\hline 1 & 01 a 33 & $-0,020$ & 5,27 & 6,64 & 0,060 & 3,49 & 6,60 \\
\hline 2 & 34 a 67 & 0,000 & 5,12 & 5,39 & 0,129 & 3,37 & 6,04 \\
\hline \multirow[t]{2}{*}{3} & 68 a 100 & $-0,029$ & 4,94 & 15,33 & 0,031 & 3,18 & 10,91 \\
\hline & Média & $-0,016$ & 5,11 & 9,12 & 0,073 & 3,35 & 7,85 \\
\hline
\end{tabular}




\section{AGRADECIMENTOS}

Ao Conselho Nacional de Desenvolvimento Científico e Tecnológico (CNPq) (bolsa de produtividade em pesquisa e IC) e à Coordenação de Aperfeiçoamento de Pessoal de Nível Superior (CAPES) (Edital 6/2012).

\section{REFERÊNCIAS}

CAMPAGNOL, R. et al. Boro e nitrogênio na incidência de hastes ocas e no rendimento de brócolis. Ciência e Agrotecnologia, v.33, p.1477-1485, 2009. Disponível em: <http://dx.doi.org/10.1590/ S1413-70542009000600004>. Acesso em: 10 jun. 2015.

CARGNELUTTI FILHO, A. et al. Tamanho ótimo de parcela em milho com comparação de dois métodos. Ciência Rural, v.41, p.1890-1898, 2011a. Disponível em: <http://dx.doi.org/10.1590/ S0103-84782011001100007>. Acesso em: 10 jun. 2015.

CARGNELUTTI FILHO, A. et al. Tamanhos de parcela e de ensaio de uniformidade em nabo forrageiro. Ciência Rural, v.41, p.1517-1525, 2011b. Disponível em: < http://dx.doi.org/10.1590/ S0103-84782011005000119>. Acesso em: 10 jun. 2015.

DINIZ, E.R. et al. Crescimento e produção de brócolis em sistema orgânico em função de doses de composto. Ciência e Agrotecnologia, v.32, p.1428-1434, 2008. Disponível em: $<$ http://dx.doi.org/10.1590/ S1413-70542008000500011>. Acesso em: 10 jun. 2015

LALLA, J.G. et al. Competição de cultivares de brócolos tipo cabeça única em Campo Grande. Horticultura Brasileira, v.28, p.360-363, 2010. Disponível em: <http://dx.doi.org/10.1590/ S0102-05362010000300020>. Acesso em: 10 jun. 2015.

MEIER, V.D.; LESSMAN, K.J. Estimation of optimum field plot shape and size testing yield in Crambe abyssinica hordnt. Crop Science, v.11, p.648-650, 1971.

MELO, R.A.C. et al. Cultivo de brócolos de inflorescência única no verão em plantio direto. Horticultura Brasileira, v.28, p.2328, 2010. Disponível em: <http://dx.doi.org/10.1590/S010205362010000100005>. Acesso em: 10 jun. 2015.

OLIVEIRA, S.J.R. et al. Plot size and experimental unit relationship in exploratory experiments. Scientia Agricola, v.62 p.585-589, 2005. Disponível em: <http://dx.doi.org/10.1590/ S0103-90162005000600012>. Acesso em: 10 jun. 2015.
PARANAÍBA, P.F. et al. Tamanho ótimo de parcelas experimentais: proposição de métodos de estimação. Revista Brasileira de Biometria, v.27, p.255-268, 2009a. Disponível em: <http://jaguar. fcav.unesp.br/RME/biometria/inicial.php>. Acesso em: 24 jun. 2014.

PARANAÍBA, P.F. et al. Tamanho ótimo de parcelas experimentais: comparação de métodos em experimentos de trigo e mandioca. Revista Brasileira de Biometria, v.27, p.8190, 2009b. Disponível em: <http://jaguar.fcav.unesp.br/RME/ biometria/inicial.php>. Acesso em: 24 jun. 2014.

PIZETTA, L.C. et al. Resposta de brócolis, couve-flor e repolho à adubação com boro em solo arenoso. Horticultura Brasileira, v.23, p.51-56, 2005. Disponível em: <http://dx.doi.org/10.1590/ S0102-05362005000100011>. Acesso em: 10 jun. 2015. doi: $10.1590 / \mathrm{S} 0102-05362005000100011$.

R DEVELOPMENT CORE TEAM. R: a language and environment for statistical computing. Vienna: R Foundation for Statistical Computing, 2013. Disponível em: $<$ http://www.R-project.org $>$.

RAMALHO, M.A.P. et al. Experimentação em genética e melhoramento de plantas. 2.ed. Lavras: UFLA, 2005. 322p.

RAMALHO, M.A.P. et al. A experimentação em genética e melhoramento de plantas. Lavras: EDUFLA, 2000. 322p.

ROSA E.A.S; RODRIGUES, A.S. Total and individual glucosinalate content in 11 broccoli cultivars grown in early and late seasons. HortScience, v.36, p.56-59, 2001. Disponível em: $<$ http://hortsci.ashspublications.org/content/36/1/56.full pdf + html $>$. Acesso em: 10 jun. 2015

STORCK, L. Partial collection of data on potato yield for experimental planning. Field Crops Research, v.121, p.286290, 2011. Disponível em: <http://dx.doi.org/10.1016/j. fcr.2010.12.018>. Acesso em: 10 jun. 2015.

STORCK, L. et al. Experimentação vegetal. 3.ed. Santa Maria: UFSM, 2011. 198p.

TREVISAN, J.N. et al. Rendimento de cultivares de brócolis semeadas em outubro na região centro do Rio Grande do Sul. Ciência Rural, v.33, p.233-239, 2003. Disponível em: <http://dx.doi.org/10.1590/ S0103-84782003000200009>. Acesso em: 10 jun. 2015.

ZIMMERMANN, F.J.P.(Ed.). Estatística aplicada à pesquisa agrícola. Santo Antonio de Goiás: Embrapa Arroz e Feijão, 2004. 402p. 